\title{
Mitotic Potential of the Enamel Organ of the Rhesus Monkey
}

\author{
W. O. ENGLER, * S. P. RAMFJORD, and J. J. HINIKER† \\ School of Dentistry, The University of Michigan, Ann Arbor, Michigan, and \\ Veterans Administration Hospital, Ann Arbor, Michigan
}

The purpose of the present investigation was to study the mitotic behavior of the cells that make up the enamel organs of developing teeth by using tritiated thymidine.

Recent radioautographic studies with tritiated thymidine have enhanced the knowledge of cell-proliferation sites and cellmigration patterns in the developing molar teeth of the guinea pig, ${ }^{1}$ rabbit, ${ }^{2}$ and $\mathrm{rat}^{3}$ and in the developing incisor of the mouse. ${ }^{4}$ In order to simulate the conditions of the developing human dentition as much as possible, rhesus monkeys were selected for this isotope study.

\section{Materials and Methods}

The same animals and techniques were used as those reported in another paper on the development of the epithelial attachment. ${ }^{b}$ Tritiated thymidine $\ddagger(1 \mu \mathrm{c} / \mathrm{gm}$ of body weight; specific activity, $6.7 \mathrm{c} / \mathrm{mmole}$ ) was administered intravenously to 1 adult monkey with a 4-month fetus and intraperitoneally to a $4 \frac{1}{2}$-month-old monkey. The animals were sacrificed 1 hour after the injection.

Only the jaws of the fetus and baby monkey were used for the present study. These were fixed in 10 per cent neutral buffered Formalin, decalcified in 20 per cent ethylenediaminetetraacetic acid, imbedded in paraffin, and sectioned at $8 \mu$ in the usual manner. Radioautographs were prepared by dipping the slides into NTB-3§ emulsion. They were exposed for 3 weeks in light-proof boxes at $4^{\circ} \mathrm{C}$., developed, and stained with hematoxylin.

Received for publication July 9, 1964.

* Present address: National Institute of Dental Research, National Institutes of Health, Bethesda, Md.

D. $\mathrm{C}^{\dagger}$

† Present address: Veterans Administration, Washington,

New England Nuclear Corporation, Boston, Mass.

\& Eastman Kodak Company, Rochester, N.Y.
Although the metabolic activity in several highly differentiated cell groups ${ }^{2,6,7}$ may induce labeling without the cells' going into division, it is generally accepted that cells which incorporate tritium-labeled thymidine are beginning DNA replication in preparation for mitosis. The labeled cells can be recognized by the localization of exposed silver grains over their nuclei. In the present investigation labeled cells will be considered indicative of impending proliferation, as has been the procedure in a number of recent studies of oral structures. ${ }^{1,3,4,8-12}$

\section{Results}

Initiation of the dental lamina from the ectoderm occurs prior to 4 months of fetal life. Thus, sections depicting the initiation of tooth development were not available. However, from the fetus and the baby monkey numerous sections demonstrated the various stages of odontogenesis following the formation of the dental lamina. The present report starts with the enamel organ at the stage of a proliferating dental lamina and extends to completed amelogenesis. The radioactive index of the fetal oral epithelium was 2.5 per cent (of 12,093 cells counted, 303 were labeled) compared with 2.0 per cent for the baby monkey. The fetal oral epithelium was 8-10 cell layers thick and showed labeling in the basal layer (Fig. 1).

In the 4-month fetus, the dental lamina appeared as a band of three to four cell layers of elongated epithelial cells and had severed its connection with the overlying oral epithelium. The lamina was located within the fetal jaw at the junction of the crypt of the deciduous tooth and the dermal connective tissue. In sections where an undifferentiated dental lamina was present, labeled cells were occasionally observed at irregular intervals. 
As bud formation occurred along the lamina, the cells lining the periphery at the site of the bud assumed a perpendicular orientation to the lamina proper and differentiated into short cuboidal cells (Fig. 2). The cells within the developing bud exhibited prickle-cell arrangement and appeared more widely spaced than the peripheral cells (Fig. 3). Many of both the outer and the inner

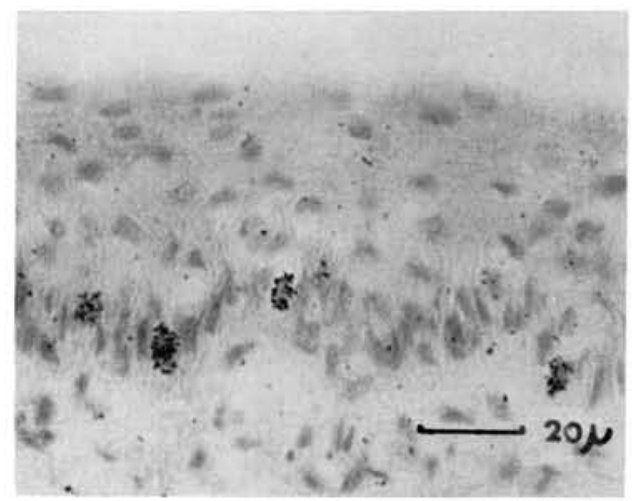

FIG. 1.-Fetal oral epithelium. Labeled cells in basal layer. (Hematoxylin stain.)

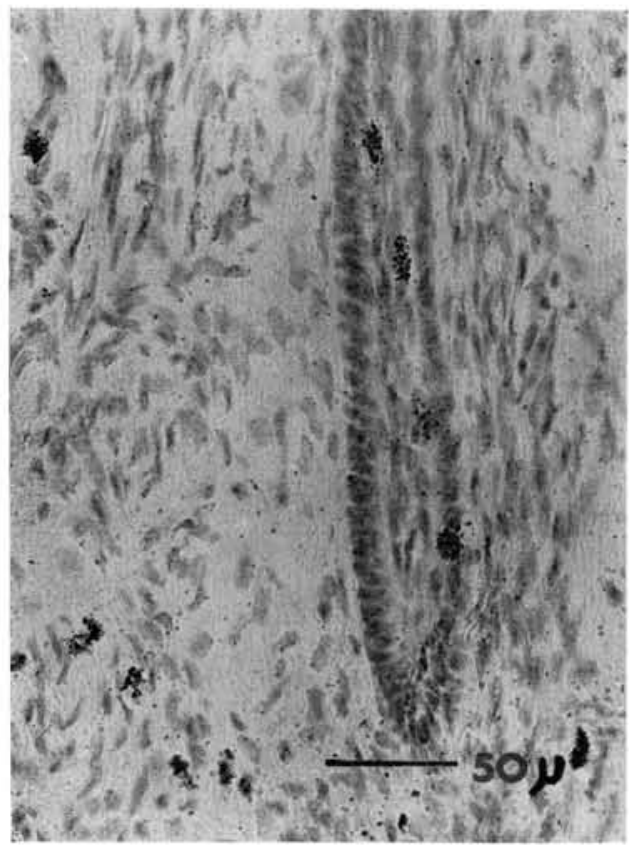

Fig. 2.-Labeled cells in outer and inner cell layers of early-bud-stage enamel organ and in surrounding connective tissue. (Hematoxylin stain.) cells of the enamel organ were labeled at this stage. Labeling was also evident in the connective tissue of the dental sack surrounding the developing bud.

Labeled cells were numerous in the epithelium of the developing enamel organ and in the surrounding connective tissue as the tooth development proceeded to the cap stage (Fig. 4). Three recognizable layers were observed in the epithelial tissues forming the cap. One outer epithelial layer composed of tightly packed cuboidal to short columnar cells covered the convexity of the cap and inclosed more widely spaced polygonal cells filling the center of the developing enamel organ. Within the concavity of the cap, the lining epithelial cells had differentiated into parallel tall columnar cells. Labeled cells were present in the epithelium along both the outer and inner borders of the cap, and some of the central cells were labeled, especially those adjacent to the inner border epithelium. Numerous labeled cells were present in the connective tissue of the dental papilla under the developing enamel organ.

As the differentiation of the epithelial cells of the enamel organ continued, the four classical layers of the bell stage were observed. At the junction of the inner and outer enamel epithelium, labeling appeared

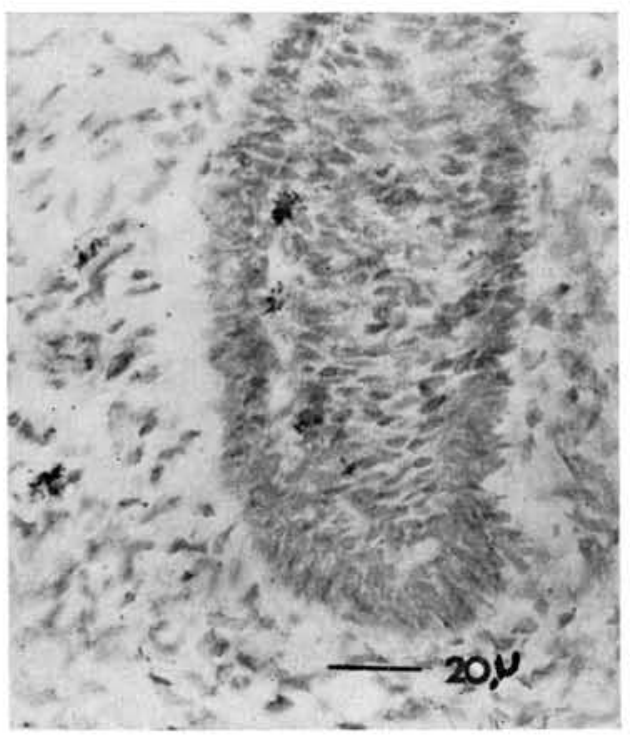

FIG. 3. - Labeled cells within center of bud-stage enamel organ of monkey fetus. (Hematoxylin stain.) 
to occur in both layers. Elsewhere, labeled cells were absent in the stellate reticulum, occasionally seen in the outer enamel epithelium, and frequently observed in the stratum intermedium and the inner enamel epithelium (Fig. 5). As the inner enamel epithelium at the future amelodentinal junction (at the tip of the cusp) developed into preameloblasts, substantial labeling could still be observed. After dentin-matrix formation was initiated, the preameloblasts in the immediate area of the newly formed dentin matrix differentiated into ameloblasts. Labeling ceased in the ameloblast layer as this differentiation occurred. The stratum intermedium was then reduced to a one-cell layer perpendicular to the ameloblasts (Fig. 6). Labeled cells were found in the stratum intermedium throughout amelogenesis. Labeling in the outer enamel epithelium was observed occasionally until completion of amelogenesis (Fig. 7).

The zone of the stellate reticulum gradually decreased in size as the enamel formation progressed until it was no longer visible as a distinct layer (Figs. 8, 9). The cells of the outer enamel epithelium and associated capillaries closely approximated the stratum intermedium at this stage (Figs. 7-10). Labeled cells still were observed in the stratum intermedium and occasionally in the outer enamel epithelial layer.
As amelogenesis was completed, the ameloblasts lost their Tomes's process configuration, and the nuclei of the ameloblasts became centrally located and stained less distinctively with hematoxylin. The stratum intermedium lost its perpendicular orientation to the reduced-ameloblast layer and formed a united nondescript layer with the cells of the outer enamel epithelium. The combined epithelial layers, that is, the inner reduced-ameloblast layer and the outer layer derived from stratum intermedium and outer enamel epithelium, together formed the reduced-enamel epithelium of the unerupted tooth. Labeling was absent in the layer of reduced ameloblasts but was occasionally seen in the outer layer of the reduced-enamel epithelium from completion of the amelogenesis until the teeth almost reached contact with the oral mucosa during eruption.

\section{Discussion}

The fetus, with its rapidly expanding cell population, provided an excellent opportunity to study cell-behavior patterns during tooth development with no interference from extraneous factors. The passage of tritiated thymidine from the mother to the fetus at 4 months' gestation was efficient since radioautographs of good quality were obtained from the fetus.

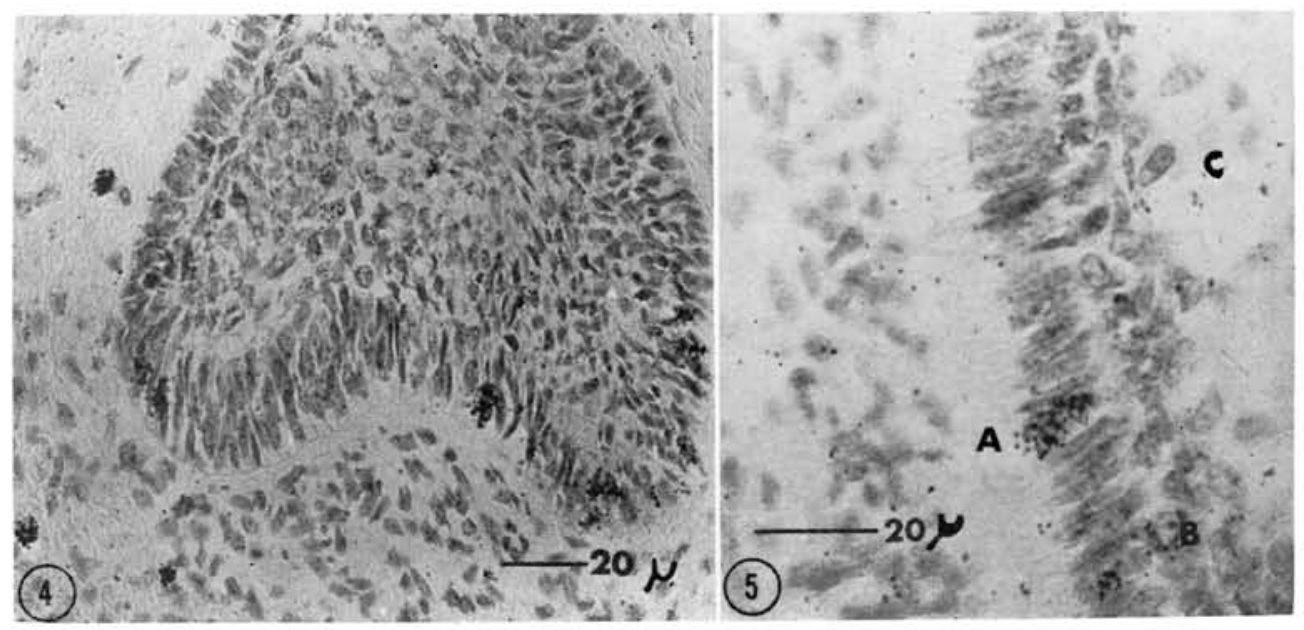

FIG. 4. - Cap-stage ename! organ of baby monkey. Labeled cells in outer and inner cell layers. (Hematoxylin stain.)

Fig. 5.- - Labeled preameloblasts $(A)$ and stratum intermedium $(B)$ in bell-stage enamel organ of baby monkey; stellate reticulum $(C)$. (Hematoxylin stain.) 
The tooth development in the monkey fetus of 4 months had progressed to the stage of deposition of enamel and dentin matrix in the crowns of the deciduous teeth and to beginning deposition of enamel-dentin matrix in the first permanent molar teeth. The Anlages of the succedaneous or permanent teeth had progressed anywhere from a growing lamina to bud, cap, and bell stages. Thus the various stages of tooth development to nearly complete amelogenesis were observed. This 4-month fetus showed

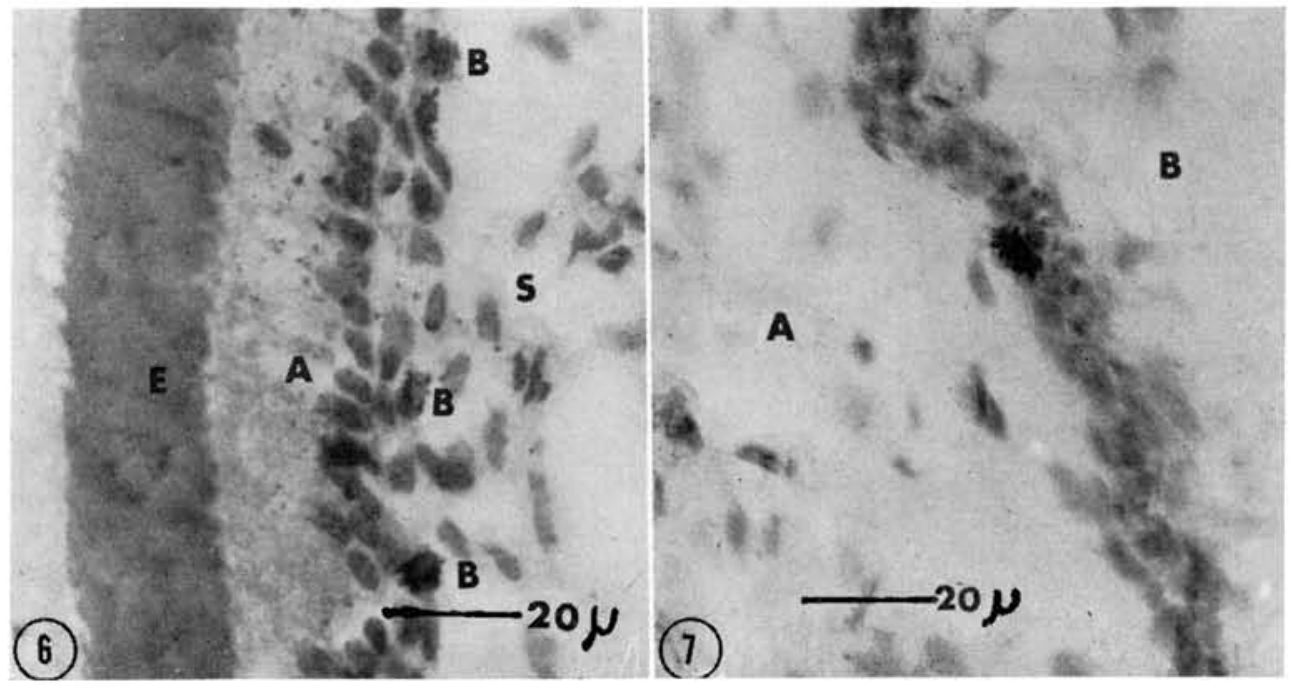

Fig. 6.-Labeled stratum-intermedium cells $(B)$ of monkey fetus. $E$, enamel matrix; $A$, ameloblasts; $S$, stellate reticulum. (Hematoxylin stain.)

FIG. 7.-Labeled cell along outer enamel epithelium of monkey fetus. $A$, stellate reticulum; $B$, connective tissue. (Hematoxylin stain.)
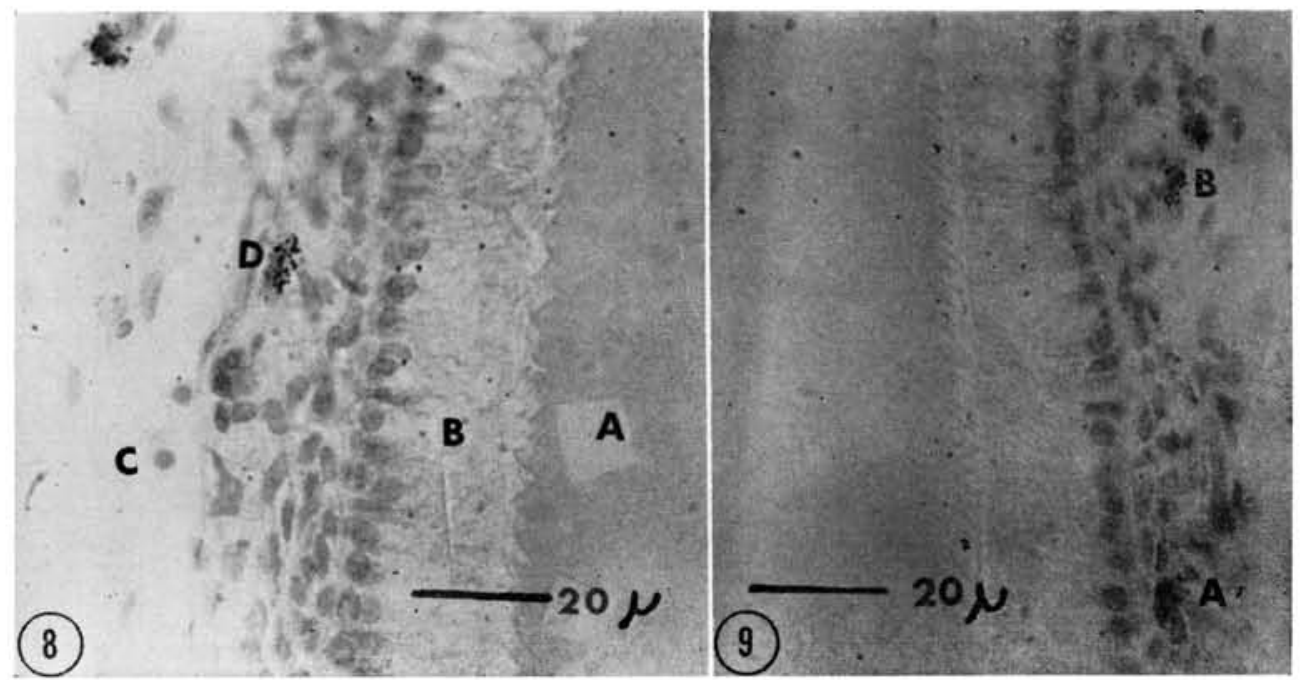

FIG. 8.-Labeled cell in outer enamel epithelium $(D)$ during advanced enamel-matrix deposition. Note absence of stellate reticulum cells. $A$, enamel matrix; $B$, ameloblasts; $C$, connective tissue. (Hematoxylin stain.)

FIG. 9.-Labeled stratum intermedium $(A)$ and outer enamel epithelium $(B)$ of monkey fetus. (Hematoxylin stain.) 
histological evidence of beginning calcification in the mandibular first permanent molars, which confirms the observation of Swindler and Gavan, ${ }^{13}$ who, from dissected specimens, have stated that calcification of the permanent dentition begins prior to birth in the rhesus monkey.

The $4 \frac{1}{2}$-month-old baby monkey exhibited permanent tooth germs from the cap stage to enamel and dentin-matrix deposition. The second deciduous molars were not erupted, but the crowns were fully formed. The crowns of the first permanent molars were approaching completion but not erupted. All the deciduous teeth except the second molars were erupted into the oral cavity, and the front teeth were in normal function. Tooth development could thus be followed from the dental lamina to the stage of functional occlusion when the slides from the fetus and the baby monkey were studied combined.

The importance of differential proliferation in the process of morphogenesis of the enamel organ has been documented previously by morphologic studies ${ }^{14-16}$ and by tritiated thymidine studies. ${ }^{1-4}$ Labeled cells were found in all layers of the enamel organ through the cap and early bell stage (Figs. $2-5)$. At the late bell stage, the stellate reticulum did not exhibit further labeling (Figs. $7-10)$. Hunt and Paynter observed in guinea pigs $^{3}$ that the only labeled cells in the stellate reticulum were along the stratum intermedium at the junction of the outer and inner enamel epithelium near the base of the enamel organ. Since the stellate reticulum of the monkey did not exhibit any labeling, it may be considered a postmitotic-cell population.

There was a marked variation in labeling of the inner enamel epithelium in the late bell stage during the formation of the enamel matrix. Undifferentiated cells of the inner enamel epithelium and preameloblasts exhibited labeling in all sections. Functioning ameloblasts, however, never exhibited labeling 1 hour after thymidine injection. It appeared that both the cells of the stellate reticulum and the mature ameloblasts lost their ability to divide as they became fully differentiated. The stellate reticulum disappeared after the crown formation was complete, and the residual non-functioning postameloblasts never showed labeling or division figures.
Labeled cells were abundant in the early stages of the stratum intermedium, when it was four to six cell layers thick. Labeling also was seen frequently during amelogenesis, when one cell layer constituted the stratum intermedium and these cells were oriented perpendicular to the ameloblasts (Figs. 5, 6, 9, 10). As the cells of the stratum intermedium lost this perpendicular orientation, only one occasional labeled cell was noted. These observations support Hunt and Paynter's ${ }^{3}$ contention that Cohen's ${ }^{17}$

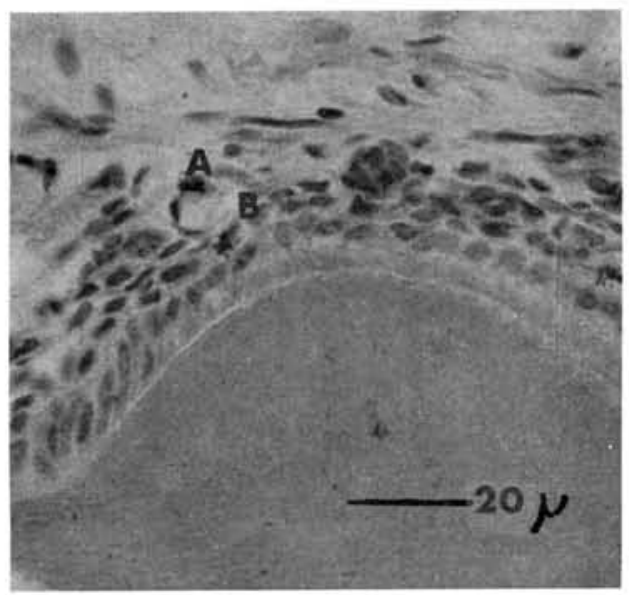

Fig. 10,-Labeled cells $(B)$ in stratum intermedium at developing cusp tip of deciduous central incisor of monkey fetus. Labeled endothelial cell (A). (Hematoxylin stain.)

hypothesis considering the reduced-enamel epithelium to be an inactive degenerating tissue is not correct. It appears that a proliferative potential is present in the stratumintermedium layer and the outer enamel epithelial layer in the monkey during amelogenesis, and these tissues could, therefore, provide protection to the underlying connective tissue, if necessary.

\section{Summary}

Mitotic activity within the enamel organs of developing teeth in a fetus and a baby rhesus monkey was studied by tritiated thymidine radioautography.

No labeling or mitotic figures were found in the stellate reticulum or in the functioning ameloblasts. Labeled cells were observed in all the other layers of the enamel organ during and following amelogenesis. It appears, therefore, that the reduced-enamel 
epithelium is not made up of degenerated cells as proposed by some investigators, since a potential for proliferation was demonstrated in these cells.

We would like to express our thanks to Miss Joan E. Wilhelm, who prepared all the radioautographs, and to Mr. Charies T. Knorpp, M.S., Assistant Chief, Radioisotope Unit, for his technical assistance. Both are from the Veterans Administration Hospital, Ann Arbor, Michigan.

\section{References}

1. Hunt, A. M., and Paynter, K. J. The Role of Cells in the Stratum Intermedium in the Development of the Guinea Pig Molar: A Study of Cell Differentiation and Migration Using Tritiated Thymidine, Arch. oral Biol., 8:65-78, 1963.

2. STARKEY, W. E. The Migration and Renewal of Tritjum Labeled Cells in the Developing Enamel Organ of Rabbits, Brit.dent. J., 115:143-53, 1963.

3. Paynter, K. J., and Hunt, A. M. Mitotic Activity in Rat Molar Tooth Germs, $f$. dent. Res., 40:652, 1961 (abstract).

4. HWANG, W. S., Tonna, E. A., and Cronkite, E. P. An Autoradiographic Analysis of the Cellular Growth of Mouse Incisors Using Tritiated Thymidine. (Abstract 141 of the 41st General Meeting of the International Association for Dental Research, 1963.)

5. Engler, W. O., Ramfjord, S. P., and Hiniker, J. J. The Development of the Epithelial Attachment and the Gingival Sulcus in Rhesus Monkeys, J. Periodont. (in press)

6. PELC, S. R. On the Question of Renewal of Differentiated Cells, Exp. Cell Res., 29:194-98, 1963.
7. Owen, M., and Macpherson, S. Cell Population Kinetics of an Oskogenic Tissue. II. (USAEC Report $B N L-6738$, Brookhaven National Laboratory, 1963.)

8. Grevitch, R. C. Epithelial DNA and RNA Synthetic Activities of the Gingival Margin, $J$. dent. Res., 40:682, 1961 (abstract).

9. - Cell Proliferation and Migration in the Epithelial Attachment Collar of the Mouse Molar. (Abstract 304,40 th General Meeting of the International Association for Dental Research, 1962.)

10. Beagrie, G. S., and SkougaARd, M. R. Observations on the Life Cycle of the Gingival Epithelial Cells of Mice as Revealed by Autoradiography, Acta odont. scand., 20:15-31, 1962.

11. Skougaard, M. R., and Beagrie, G. S. The Renewal of Gingival Epithelium in Marmosets (Callithrix jacchus) as Determined through Autoradiography with Thymidine $\mathrm{H}^{3}$, Acto odont. scand., 20 : 467-84, 1962.

12. Drmassimo, C. Proliferation and Migration of Cells in the Gingival Epithelium, pp. iii, 58. Unpublished M.S. thesis, Eastman Dental Dispensary, Rochester, New York, 1963.

13. Swindler, D. R., and Gavan, J. A. Calcification of the Mandibular Molars in Rhesus Monkeys, Arch. oral Biol., $7: 727-34,1962$.

14. Schour, I., and MAssler, M. Studies in Tooth Development: The Growth Pattern of Human Teeth. I, J. Amer. dent. Ass., 27:1778-93, 1940.

15. WAKEFORD, C. A. Some Observations on the Tooth Band and on the Enamel Organs of the Human Deciduous Teeth, Proc. roy. Soc. Med. (Odoniol. Sec.), 20:13-28, 1927.

16. LeFKowitz, W., BODECKER, C. F., and MARDFIN, D. E. Odontogenesis of the Rat Molar: Prenatal Stage, J. dent. Res., 32:749, 1953 (abstract).

17. Coren, B. Pathology of Interdental Tissues, Dent. Practit. dent. Rec., 9:167-73, 1959. 